\title{
A Socio-technical Approach to Pre- venting, Mitigating, and Recovering from Ransomware Attacks
}

\author{
Dean F. Sittig ; Hardeep Singh ${ }^{2,3}$ \\ ${ }^{1}$ University of Texas Health Science Center at Houston, School of Biomedical Informatics and UT-Memorial Hermann Center for \\ Health Care Quality and Safety, Houston, Texas; \\ ${ }^{2}$ Houston Veterans Affairs Center for Innovations in Quality, Effectiveness and Safety, Michael E. DeBakey Veterans Affairs Medi- \\ cal Center, Houston, Texas; \\ ${ }^{3}$ Section of Health Services Research, Department of Medicine, Baylor College of Medicine, Houston, Texas
}

\section{Keywords}

Health information technology, electronic health record, socio-technical, cybersecurity, ransomware

\begin{abstract}
Summary
Recently there have been several high-profile ransomware attacks involving hospitals around the world. Ransomware is intended to damage or disable a user's computer unless the user makes a payment. Once the attack has been launched, users have three options: 1) try to restore their data from backup; 2) pay the ransom; or 3) lose their data. In this manuscript, we discuss a socio-technical approach to address ransomware and outline four overarching steps that organizations can undertake to secure an electronic health record (EHR) system and the underlying computing infrastructure. First, health IT professionals need to ensure adequate system protection by correctly installing and configuring computers and networks that connect them. Next, the health care organizations need to ensure more reliable system defense by implementing user-focused strategies, including simulation and training on correct and complete use of computers and network applications. Concomitantly, the organization needs to monitor computer and application use continuously in an effort to detect suspicious activities and identify and address security problems before they cause harm. Finally, organizations need to respond adequately to and recover quickly from ransomware attacks and take actions to prevent them in future. We also elaborate on recommendations from other authoritative sources, including the National Institute of Standards and Technology (NIST). Similar to approaches to address other complex socio-technical health IT challenges, the responsibility of preventing, mitigating, and recovering from these attacks is shared between health IT professionals and end-users.
\end{abstract}

\section{Correspondence to:}

Dean F. Sittig, PhD

The University of Texas Health Science Center at Houston (UTHealth)

School of Biomedical Informatics

UT-Memorial Hermann Center for Healthcare Quality \&

Safety

6410 Fannin St.

UTPB 1100.43

Houston, TX 77030

Email: Dean.F.Sittig@uth.tmc.edu
Appl Clin Inform 2016; 7: 624-632

http://dx.doi.org/10.4338/ACI-2016-04-SOA-0064

received: April 25, 2016

accepted: June 13, 2016

published: June 29, 2016

Citation: Sittig DF, Singh H. A socio-technical approach to preventing, mitigating, and recovering from ransomware attacks. Appl Clin Inform 2016; 7: 624-632

http://dx.doi.org/10.4338/ACI-2016-04-SOA-0064

Funding

Dr. Singh's research is supported by the VA Health Services Research and Development Service (CRE 12-033; Presidential Early Career Award for Scientists and Engineers USA 14-274), the VA National Center for Patient Safety, the Agency for Health Care Research and Quality (R01HS022087 and R21 HS 023602) and in part by the Houston VA HSR\&D Center for Innovations in Quality, Effectiveness and Safety (CIN 13-413). 


\section{Introduction}

Rapid adoption of electronic health records (EHRs) has fundamentally changed the way health care organizations and clinicians care for patients, manage the hospital, account for health care quality, and bill for their services. Recently there have been several high-profile ransomware attacks involving hospitals [1-6]. Furthermore, a recent survey of 61 chief information officers, chief information security officers, and other IT director-level respondents conducted by HIMSS Analytics (Chicago, IL) found that more than half of them had been targets of ransomware attacks in the previous 12 months [7]. Most of these organizations either a) fended off the attacks through intelligent use of network and user activity surveillance systems, b) were able to restore their critical systems from backups, or c) quietly paid the ransom. Reports of these events are generally leaked to the news media only after hospital operations are compromised for an extended period of time. In the absence of a centralized learning system for these events [8], it is not possible to decipher specific details of what happened, how it was initiated, who was responsible, and how it was resolved.

While specific details of how ransomware attacks begin are not well known, they often start when a user is tricked into clicking a link or opening an attachment of a malicious email message. Software that is intended to damage or disable the computer is then downloaded to the user's computer, and it quickly encrypts all of the data on that machine and possibly reaches out over the network to encrypt data on other machines as well, thus rendering all data inaccessible [9]. The user is then presented a message stating that all the files have been encrypted, and if they do not pay a ransom within a short period of time, the files will be destroyed. Once the attack has been launched, users have three basic options: 1) try to restore their data from a backup; 2) pay the ransom; or 3) lose their data. The goal of this paper is to provide recommendations to health care organizations (HCOs) on how to prevent and mitigate these malicious events. We use a socio-technical approach to address ransomware and outline four overarching steps that organizations can undertake to secure an EHR and the underlying computing infrastructure.

\section{Origin of Ransomware}

While ransomware in hospitals seems to be much discussed these days, the concept dates back to the distribution of the "AIDS Trojan virus" via floppy disk through surface mail back in 1989 [10]. Over fifteen years of internet revolution passed before the next instance of ransomware (GPCoder), which was delivered via e-mail in 2005 [11, 12]. The means of distribution of these ransomware programs has since grown to include malicious advertisements; USB drives; macros embedded in documents, spreadsheets, and presentations; archived files; batch or command files; and executables. The means of paying the ransom has also evolved from sending checks to off-shore bank accounts to paying via PayPal, from requiring users to purchase cash cards from certain websites, to paying with bitcoins. With increasing electronic data, malicious programs that encrypt key files and demand payment for the decryption key must now be taken seriously [13]. Moreover, health care has become more electronic than ever, making it attractive to hackers.

\section{Conceptual Approach to Addressing Health IT Ransomware}

As with most health information technology-related (HIT) issues, preventing a ransomware attack is a complex socio-technical problem [14]. For example, ransomware attacks often rely on some form of "social engineering," or the psychological manipulation of people in an attempt to gain their trust and lead them to divulge confidential information, along with a sophisticated encryption algorithm (i.e., technical part of problem). Solving these types of socio-technical problems is a shared task between those responsible for configuring, maintaining, and operating the organization's HIT infrastructure as well as the users of this infrastructure. While preventing all ransomware attacks is not possible, there are a number of steps HCOs can take to reduce their risk as well as mitigate potential harm. 
Based on previously developed health IT-related conceptual frameworks and the National Institute of Standards and Technology (NIST) Framework for Improving Critical Infrastructure Cybersecurity [15], we outline four overarching socio-technical steps to secure an EHR system and the underlying computing infrastructure $[16,17]$. First, health IT professionals need to ensure adequate system protection by correctly installing and configuring computers and networks that connect them. Next, HCOs need to ensure more reliable system defense by implementing user-focused strategies, including simulation and training on correct and complete use of computers and network applications. Concomitantly, the organization needs to monitor computer and application use continuously in an effort to detect suspicious activities and identify and address security problems before they cause harm. Finally, organizations need to respond adequately to and recover quickly from a ransomware attack and take actions to prevent them in future.

In the sections below, we outline a comprehensive, multi-faceted socio-technical approach to preventing and mitigating these attacks. The detailed recommendations follow Sittig and Singh's eight dimensional socio-technical model and are summarized in $>$ Table 1 . These recommendations address all five functions of the NIST Cybersecurity Framework Core - Identify, Protect, Detect, Respond, Recover, thus providing HCOs with an operational strategy for management of ransomware risk. While some of the recommendations we suggest might be common-sense and obvious, often these mishaps occur from failing to adhere to the most basic recommendations. For example, the most likely cause of the attack on MedStar was "an improperly installed JBoss server" that "appears to have used the default settings leaving access to the server's management interface open to the Internet" [18]. Even after the attack, many institutions remain vulnerable, as shown by an Internet scan revealing that 2.1 million systems still remain vulnerable to the same JBoss exploit used in the attack [19]. In addition, several of our recommendations, synthesized from a variety of sources, map directly to the Health Insurance Portability and Privacy Act (HIPAA) Security rule [20].

\section{Step 1 - Ensure Adequate System Protection by Correctly Installing and Configuring Computers and Networks}

The computing infrastructure must be prepared for a ransomware attack by creating a regular backup process for the data. This backup should be made frequently (i.e., at least daily, and a continuous or real-time backup is ideal). Copies of these backups should be stored offline to ensure that ransomware has no access to them. In addition, organizations should maintain a "gold image" of system configurations (i.e., one that allows an organization to reset systems to the pre-attack state). One should also test the organization's ability to restore these backups on a regular basis (e.g., quarterly for key data resources, yearly for less important aspects of the system).

Personnel in the organization responsible for maintaining all of the computers' operating systems, application software, browsers and plug-ins, firmware, and anti-virus software should ensure that they are up-to-date with the latest patches. Before applying any patches, health IT professionals should thoroughly test them, along with the rest of the technical and application infrastructure, to ensure that the patches do not create new, unforeseen problems. Network engineers should also ensure that the organization's firewall is properly configured (e.g., require passwords on Remote Desktop Protocol [RDP] ports), to prevent unauthorized people or programs from accessing missioncritical organizational resources. It may be necessary to segment the network by categorizing IT assets (e.g., desktops, servers, routers), data, and personnel into groups and restricting access to these groups using entry and exit traffic filtering. Finally, at the local device level, organizations should consider disabling USB (Universal Serial Bus) ports to prevent malicious software delivery [21].

In addition to these hardware and software-specific precautions, organizations should develop a "whitelist" of specified programs that are allowed to run, while blocking all others in order to prevent malicious executables from running. Furthermore, the organization should consider blocking email messages with potentially weaponized attachments ( [note: this is not an exhaustive list] ${ }^{*}$.exe,

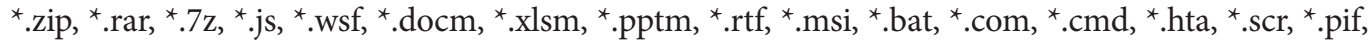
${ }^{\star}$.reg, ${ }^{\star} . \mathrm{vbs},{ }^{*} . \mathrm{cpl},{ }^{\star}$.jar files), from suspicious or unknown sources (e.g., sitwithheprop.ru or xxxiooo.com) $[22,23]$. 
Organizations should also consider restricting the ability of users to "write" (i.e., create and delete files), on shared drives of departmental or group shares. They should also consider limiting users' ability to install and run software applications using the principle of "Least Privilege," or minimize users' access to only those systems and services required by their job. This may include restricting users' administrative privileges on local desktops and laptops. For users who require administrative access, configure two accounts, one with administrative privileges that is used only when necessary, and one with restricted privileges (e.g., no ability to install new applications), that they use for routine activities, including reading email and browsing the Internet.

\section{Step 2 - Ensure More Reliable System Defense by Implementing User- Focused Strategies}

Once all the computers and networks are installed and configured appropriately, the next line of defense is adequate training so that users correctly operate their devices and applications. Additionally, health IT professionals should review organization-wide electronic messages to ensure they conform to criteria for "legitimacy" below. Health care organizations do not have to develop their own training courses for either their end-users or health IT professionals; many commercially available courses exist [24].

IT professionals must help create messages such that users can easily recognize them as legitimate e-mails. Specifically, legitimate messages from one's own institution (e.g., employer's IT department), should not ask users to download and run file attachments or ask them to enter account or password information. In addition, these messages should have a recognizable telephone number that can be cross-referenced in the local directory to enable an out-of-band check, or a personal email address with a legitimate user name that can be cross-referenced in the local directory. All email and website links should display the complete internet address (URL) to build trust.

End-users should be instructed on how to approach unrecognized emails with links and attachments. An example of such an approach is as follows:

- First Hover - on the link with your mouse pointer to identify where the link is taking you.

- Take a Second to Think - Any link, or attachment that is not from your own organization, or a recognized friend, should not to be clicked. When in doubt, either call or email (in a separate email) your friend or the organization requesting information to confirm it is legitimate.

- Only When Sure, Click

In addition to making end-users aware about the risks and proper responses to fraudulent email messages with attachments, health IT professionals should conduct simulated phishing attacks by sending fake (but safe) email messages or links to websites that appear to be from legitimate sources $[25,26]$. They should also increase their ability to respond to a successful ransomware attack by periodically conducting mock system recovery exercises (i.e., identify backups and test restore capabilities).

Although this might be the norm at some places, all health IT departments should configure their virus protection software to scan all software downloaded from the internet prior to allowing users to execute it. They should also conduct regular risk and business impact assessments to identify key applications and data based on importance to the business (e.g., Tier 0 - essential for business operations; Tier 1 - 1 hour downtime acceptable; Tier 2 - 1 day downtime acceptable; Tier 3 - 1 week downtime acceptable). This could help develop a plan to manage a ransomware attack. Finally, the organization should require 2 -factor authentication (i.e., something you have - token or cellphone, coupled with something you know - password), for remote access to applications.

\section{Step 3 - Ensure Comprehensive System Monitoring of Suspicious Activ- ities}

All organizations should develop a network and user activity monitoring system that conducts surveillance for suspicious activities (e.g., similar to the anomaly detection algorithms that credit card 
companies use to identify stolen cards) [27], such as receipt of email messages from known fraudulent sources, executable email attachments, unexpected changes in key files on network-attached drives, unknown processes encrypting files, or significant increases in network traffic on unexpected ports.

The organization should also continuously monitor the external environment for new security incidents (i.e., zero-day exploits, an attack that takes advantage of a security vulnerability on the same day that the vulnerability becomes generally known) [28], and address gaps and deficiencies as they are identified.

\section{Step 4 - Respond, Recover, Investigate, and Learn from Ransomware Attacks}

Often the first indication that a ransomware attack has occurred is an alarming message sent to the user's desktop background, or a window opens to a ransomware program that the user cannot close which contains instructions on how to pay the ransom. In these cases, users should turn off the computer and report it to their IT support team immediately. The IT professionals should disconnect the infected computer(s) from the network and turn off wireless network functionality of the infected machine. If the attack is widespread, the IT department should shut down all network operations (i.e., both wired and wireless), to prevent the malware from spreading.

Once the immediate threat is contained, the IT department should contact their organization's insurance provider, a computer forensics expert, and in the USA, the Federal Bureau of Investigation's (FBI) Internet Crime Complaint Center (http://www.ic3.gov/default.aspx). In addition, the organization should consider using an organization-wide password reset after recovery (i.e., immediately require all users to reset their passwords).

Following any unexpected extended system downtimes, whether caused by ransomware or some other human or naturally occurring event, the organization should convene a multi-disciplinary investigation team consisting of key administrative and clinical stakeholders and Health IT professionals [29] to review the event and its management, identify potential root causes, and discuss future prevention or mitigating procedures [30]. The organization should also consider consulting with external experts in IT system reliability to review and report on recommendations for improvements in key system components, configurations, and policies and procedures [31].

\section{Conclusions}

With the recent rapid adoption of EHRs, the threat of ransomware in health care facilities is greater than ever. Simply sending an email message to all employees reminding them not to click on suspicious links or attachments in email messages is no longer sufficient to prevent the emerging threat of cyber-crime in the current, fast-paced, clinical computing environment. We outline a socio-technical approach to address ransomware that involves four overarching steps that health care organizations can undertake to secure an EHR and the underlying computing infrastructure. Similar to approaches to address other complex socio-technical health IT challenges, the responsibility of preventing, mitigating, and recovering from these attacks is shared between health IT professionals and end-users.

\section{Acknowledgements}

The authors thank Soumitra Sengupta, PhD and Amar Yousif, BSEE, MBA, CISSP, GCFA for their comments on an early draft of this manuscript. 
Table 1 An Eight Dimensional Socio-technical Approach for Preventing or Mitigating Ransomware Attacks. (Based on Sittig \& Singh's Eight Dimensional Socio-technical model) [32]

\begin{tabular}{|c|c|}
\hline $\begin{array}{l}\text { Socio-techni- } \\
\text { cal dimension }\end{array}$ & Recommendations for Health Care Organizations \\
\hline $\begin{array}{l}\text { Hardware/Soft- } \\
\text { ware }\end{array}$ & $\begin{array}{l}\text { - Perform regular backups of your data. Be sure to back up frequently (continuous or real- } \\
\text { time backup may be ideal), and store your backups offline } \\
\text { - Maintain a "gold image" of system configurations (i.e., allows an organization to reset sys- } \\
\text { tems to the pre-attack state) } \\
\text { - Test your backup's restore function regularly (e.g., quarterly for key data resources, yearly } \\
\text { for less important aspects of the system) } \\
\text { - Keep your operating system, application software, browsers and plug-ins, firmware, and } \\
\text { anti-virus software up-to-date with the latest patches } \\
\text { - Make sure your firewall is properly configured (e.g., require passwords on Remote Desktop } \\
\text { Protocol [RDP] ports) } \\
\text { - Segment your network by categorizing IT assets (e.g., desktops, servers, routers), data, and } \\
\text { personnel into groups, and restricting access to these groups using entry and exit traffic fil- } \\
\text { tering } \\
\text { - Consider disabling USB (Universal Serial Bus) ports to prevent malicious software delivery } \\
\text { - Following a successful attack, disconnect the infected computers from the network } \\
\text { - Turn off wireless network functionality of the infected machine } \\
\text { - If the attack is widespread, shut down all network operations to prevent the malware from } \\
\text { spreading }\end{array}$ \\
\hline Clinical Content & 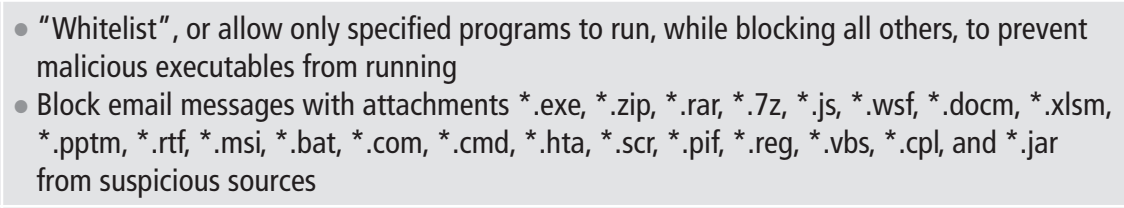 \\
\hline User Interface & $\begin{array}{l}\text { - Legitimate messages should have a telephone number someone can call (i.e., out of band } \\
\text { check), and a personal email address which has a legitimate user name that people can } \\
\text { check in their local directory; email and website links should display complete internet ad- } \\
\text { dress (URL) to build trust } \\
\text { Often the first indication that an attack has occurred is an alarming message sent to the } \\
\text { desktop background, or a window opens to a ransomware program that you cannot close, } \\
\text { with instructions on how to pay the ransom; users should turn off the computer and report } \\
\text { it to their IT support team immediately }\end{array}$ \\
\hline People & 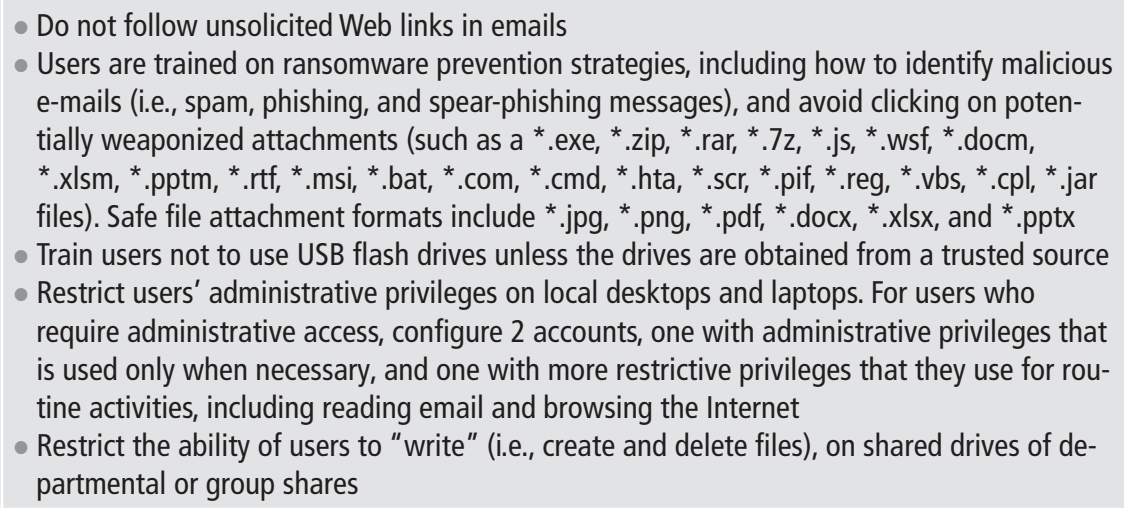 \\
\hline $\begin{array}{l}\text { Workflow/com- } \\
\text { munication }\end{array}$ & $\begin{array}{l}\text { - Scan all software downloaded from the internet prior to executing it } \\
\text { - Conduct simulated phishing attacks (i.e., fraudulent email messages or websites that ap- } \\
\text { pear to be from legitimate sources), to raise user's awareness of the problem } \\
\text { - Conduct mock system recovery exercises (i.e., identify backups and test restore capabilities) } \\
\text { - Conduct regular risk assessments } \\
\text { - Require 2-factor authentication for remote access to applications } \\
\text { - Consider using organization-wide password reset (expiration) in response to a successful } \\
\text { attack }\end{array}$ \\
\hline
\end{tabular}


Table 1 Continued

\begin{tabular}{|c|c|}
\hline $\begin{array}{l}\text { Socio-techni- } \\
\text { cal dimension }\end{array}$ & Recommendations for Health Care Organizations \\
\hline $\begin{array}{l}\text { es, and } \\
\text { ent }\end{array}$ & $\begin{array}{l}\text { - Based on risk and business impact assessments, identify applications and data based on } \\
\text { importance to the business (e.g., Tier } 0 \text { - essential for business operations; Tier } 1 \text { - } 1 \text { hour } \\
\text { downtime acceptable; Tier } 2-1 \text { day downtime acceptable; Tier } 3-1 \text { week downtime ac- } \\
\text { ceptable) - Develop a plan to manage a ransomware situation accordingly } \\
\text { - Restrict users' ability to install and run software applications using the principle of "Least } \\
\text { Privilege," or minimize users' access to only those systems and services required by their } \\
\text { job } \\
\text { - IT security should be under the control of executives with extensive IT experience (e.g., CIO } \\
\text { or Chief Information Security Officer) } \\
\text { - Consider blocking users' access to personal email accounts (e.g., Gmail, Yahoo, Hotmail, } \\
\text { iCloud) and web advertisements to avoid malvertising (i.e., insertion of malicious code into } \\
\text { online advertisements to infect unsuspecting users) }\end{array}$ \\
\hline $\begin{array}{l}\text { les } \\
\text { tions }\end{array}$ & $\begin{array}{l}\text { - Review all information security-related HIPAA requirements } \\
\text { - Contact your organization's insurance provider, a computer forensics expert, and the FBI in } \\
\text { the event of a successful attack }\end{array}$ \\
\hline $\begin{array}{l}\text { Measurement } \\
\text { and Monitoring }\end{array}$ & $\begin{array}{l}\text { - Monitor network activity to identify suspicious activity } \\
\text { - Monitor the external environment for security incidents and address gaps and deficiencies } \\
\text { - Following unexpected extended system downtime (e.g., ransomware), convene an investi- } \\
\text { gation team consisting of key stakeholders and Health IT professionals to review the event } \\
\text { and its management, identify potential root causes, and discuss future prevention or miti- } \\
\text { gating procedures }\end{array}$ \\
\hline
\end{tabular}




\section{References}

1. Kandel J and Kovacik R. Hollywood Hospital 'Victim of Cyber Attack'. NBC4 News. February 12, 2016. Available at: http://www.nbclosangeles.com/news/local/Hollywood-Hospital-Victim-of-Cyber-At tack-368574071.html

2. Steffen S. Hackers hold German hospital data hostage. Made for Minds. 25 February 2016. Available at: http://dw.com/p/1I2Xu

3. Olenick D. The Ottawa Hospital fends off ransomware attack. SC Magazine. 14 March 2016. Available at: http://www.scmagazine.com/the-ottawa-hospital-fends-off-ransomware-attack/article/482921/

4. Krebs B. Hospital Declares 'Internal State of Emergency' After Ransomware Infection. Krebsonsecurity.com. March 16, 2016. Available at: http://krebsonsecurity.com/2016/03/hospital-declares-internetstate-of-emergency-after-ransomware-infection/

5. Simms B. FBI investigating computer virus at MedStar Health. WBALTV11.com March 29, 2016. Available at: http://www.wbaltv.com/news/fbi-investigating-computer-virus-at-medstar-health/38731548

6. Gallagher S. Two more healthcare networks caught up in outbreak of hospital ransomware. Ars Technica. March 29, 2016. Available at: http://arstechnica.com/security/2016/03/two-more-healthcare-networkscaught-up-in-outbreak-of-hospital-ransomware/

7. Sullivan T. More than half of hospitals hit with ransomware in last 12 months. Health IT News. April 07, 2016. Available at: http://www.healthcareitnews.com/news/more-half-hospitals-hit-ransomware-last12-months

8. Sittig DF, Classen DC, Singh H. Patient safety goals for the proposed Federal Health Information Technology Safety Center. J Am Med Inform Assoc 2015; 22(2): 472-478. doi: 10.1136/amiajnl-2014-002988.

9. Fischer T. Private and Public Key Cryptography and Ransomware. Center for Internet Security (CIS). December 2014. Available at: https://msisac.cisecurity.org/whitepaper/documents/10.pdf

10. Gazet A. Comparative analysis of various ransomware virii. Journal in computer virology. 2010 Feb 1;6(1):77-90.

11.Mun J. Trojan.Gpcoder. Symantec. May 22, 2005. Available at: https://www.symantec.com/security_re sponse/writeup.jsp?docid=2005-052215-5723-99

12. Giri BN, Jyoti N. The Emergence of Ransomware. 9th Annual Association of anti-Virus Asia Researchers (AVAR) International Conference - Digital Security: Prevention to Prosecution. Auckland, NZ. 2006. Available at: http://citeseerx.ist.psu.edu/viewdoc/download?doi=10.1.1.169.5881\&rep=rep1\&type=pdf

13.Largent W. Ransomware: Past, Present, and Future. April 11, 2016. Available at: http://blog.talosintel.com/2016/04/ransomware.html

14. United States Computer Emergency Readiness Team. Alert (TA16-091A): Ransomware and Recent Variants. Original release date: March 31, 2016. Available at: https://www.us-cert.gov/ncas/alerts/TA16-091A

15. National Institute of Standards and Technology. Framework for Improving Critical Infrastructure Cybersecurity Version 1.0 February 12, 2014. Available at: http://www.nist.gov/cyberframework/upload/cy bersecurity-framework-021214.pdf

16.Sittig DF, Singh H. Electronic health records and national patient-safety goals. N Engl J Med 2012; 367(19): 1854-1860. doi: 10.1056/NEJMsb1205420.

17. Singh H, Sittig DF. Measuring and improving patient safety through health information technology: The Health IT Safety Framework. BMJ Qual Saf 2016; 25(4): 226-232. doi: 10.1136/bmjqs-2015-004486.

18. Gallagher S. Maryland hospital: Ransomware success wasn't IT department's fault: MedStar denies ransom payment, denies earlier JBoss bugs played role. Ars Technica. Apr 7, 2016. Available at: http://arstechnica.com/security/2016/04/maryland-hospital-group-denies-ignored-warnings-allowed-ransomware-attack/

19. Gallagher S. Maryland hospital group hit by ransomware launched from within [Updated] Samsam malware injected into network from exploited web app server at MedStar. Ars Technica. March 31, 2016. Available at: http://arstechnica.com/security/2016/03/maryland-hospital-group-hit-by-ransomware/

20.DHHS Office for Civil Rights. HIPAA Security Rule Crosswalk to NIST Cybersecurity Framework. Available at: http://www.hhs.gov/sites/default/files/NIST\%20CSF\%20to\%20HIPAA\%20Secur ity\%20Rule\%20Crosswalk\%2002-22-2016\%20Final.pdf

21. Wright A, Sittig DF. Security threat posed by USB-based personal health records. Ann Intern Med. 2007 Feb 20;146(4):314-5.

22.Hoffman C. How To Spot A Dangerous Email Attachment. 20 Jan 2014. Available at: http://www.make useof.com/tag/spot-dangerous-email-attachment/)

23.DNS-BH - Malware Domain Blocklist. Available at: http://www.malwaredomains.com/

24.Protection of Information Assets: CISA Tutorial. Available at: http://www.simplilearn.com/protection-ofinformation-assets-cisa-tutorial-video 
25.Scarfone K, Souppaya M, Cody A, Orebaugh A. Technical Guide to Information Security Testing and Assessment: Recommendations of the National Institute of Standards and Technology. NIST Special Publication 800-115. September 2008. Available at: http://csrc.nist.gov/publications/nistpubs/ 800-115/SP800-115.pdf

26. Youngstrom N. Hospital Uses Fake Phishing Emails in Security Training; Will Move to Gamification. Report on Medicare Compliance. 25: 17; May 9, 2016.

27. Van Vlasselaer V, Bravo C, Caelen O, Eliassi-Rad T, Akoglu L, Snoeck M, Baesens B. APATE: A novel approach for automated credit card transaction fraud detection using network-based extensions. Decision Support Systems 2015; 75: 38-48.

28. Bilge L, Dumitras T. Before we knew it: an empirical study of zero-day attacks in the real world. In Proceedings of the 2012 ACM conference on Computer and communications security. 2012 Oct 16 (pp. 833-844).

29. Singh H, Classen DC, Sittig DF. Creating an oversight infrastructure for electronic health record-related patient safety hazards. J Patient Saf 2011; 7(4): 169-174. doi: 10.1097/PTS.0b013e31823d8df0.

30.Sittig DF, Gonzalez D, Singh H. Contingency planning for electronic health record-based care continuity: a survey of recommended practices. Int J Med Inform 2014; 83(11): 797-804. doi: 10.1016/j.ijmedinf.2014.07.007.

31.Siwicki B. Tips for protecting hospitals from ransomware as cyberattacks surge. HealthIT News. April 6, 2016. Available at: http://www.healthcareitnews.com/news/tips-protecting-hospitals-ransomware-cyberattacks-surge

32. Sittig DF, Singh H. A new sociotechnical model for studying health information technology in complex adaptive healthcare systems. Quality and Safety in Health Care 2010; 19(Suppl. 3): i68-i74. 